\title{
Eventos adversos asociados al uso de los antibióticos claritromicina y azitromicina
}

Adverse events associated with the use of the antibiotics clarithromycin and azithromycin
Eventos adversos associados ao uso de antibióticos claritromicina e azitromicina
Fecha de Recepción

10 de octubre de 2018
Aceptado para su publicación

16 de noviembre de 2018
María T Rocha

Profesora Adjunta. Cátedra de Farmacología.

Email:trissirocha@gmail.com

Sergio D Morales

Jefe de Trabajos Prácticos.

Cátedra de Farmacología.

Email: morales.sergiodaniel500@gmail.com

Isabel Hartman

lefe de Trabajos Prácticos.

Cátedra de Farmacología.

Email: hartmanisabel@gmail.com

Mauricio Schmidt

Jefe de Trabajos Prácticos.

Práctica Final Obligatoria.

Email:drmauri@yahoo.com.ar

Roxana Servín

Jefe de Trabajos Prácticos.

Práctica Final Obligatoria.

Email: roxivida@hotmail.com

Lorena Dos Santos

Profesora Titular. Cátedra de Farmacología.

Email: lorenatirabosco@gmail.com

Facultad de Medicina. Universidad Nacional del Nordeste. Moreno I 240 (3400) Corrientes, Argentina. Tel: +5493794432902 .
Resumen

En Odontología se prescriben antibióticos de manera empírica para el tratamiento de procesos infecciosos, siendo los macrólidos una alternativa terapéutica para casos de hipersensibilidad y resistencia bacteriana. Sin embargo, su uso no está exento de riesgos. El objetivo de este estudio fue identificar y caracterizar los eventos adversos asociados al uso de claritromicina y azitromicina. Se realizó un estudio observacional-descriptivo- transversal de Farmacovigilancia Proactiva en pacientes de dos Centros de Salud de la Ciudad de Corrientes que recibían antibióticos macrólidos, azitromicina o claritromicina, durante un período de dieciocho meses. Variables analizadas: edad, sexo, tratamiento farmacológico actual según diagnóstico, características de los eventos adversos identificados según: órganos y tejidos afectados; gravedad, tipo de reacción (A ○ B según mecanismo de producción); imputabilidad y resultado de la reacción (recuperado, recuperado con secuelas, no recuperado, desconocido, riesgo de vida). Se registraron 97 pacientes, de los cuales $26(27 \%)$ presentaron eventos adversos. Los órganos afectados con mayor frecuencia fueron el sistema gastrointestinal (14, $54 \%$ ) y cardiovascular en segundo término (5, $19 \%)$. Tres fueron graves (11\%) y requirieron suspender la medicación, 7 moderados (27\%) y 16 leves (62\%). El 100\% se recuperaron ad integrum. Según el mecanismo de producción 22 (85\%) fueron tipo A y 4 (15\%) de tipo B. Según 
REVISTA FACULTAD DE ODONTOLOGIAA

ISSN No 1668-7280 - Vol. XI No 1 - 2018

la imputabilidad I 8 (82\%) fueron probables y 3 probadas (12\%). Los datos obtenidos muestran un potencial de eventos cardiovasculares, cuya gravedad debiera ser tenida en cuenta para la toma de decisiones relacionadas con la práctica clínica odontológica a fin de evitar un uso innecesario.

\section{Palabras clave}

Efectos Colaterales y Reacciones Adversas Relacionados con Medicamentos. Azitromicina. Claritromicina.

\section{Summary}

In Dentistry, antibiotics are prescribed empirically for the treatment of infectious processes, being the macrolides a therapeutic alternative for cases of hypersensitivity and bacterial resistance. However, its use is not free of risks. The objective of this study was to identify and characterize the adverse events associated with the use of clarithromycin and azithromycin. An observational-descriptive-cross-sectional study of Proactive Pharmacovigilance was performed in patients, from two Health Centers in the City of Corrientes, who received macrolide antibiotics as azithromycin or clarithromycin during a period of eighteen months. Variables analyzed: age, sex, current pharmacological treatment according to diagnosis, adverse events characteristics identified according to: affected organs and tissues; severity, type of reaction (A or B according to the mechanism of production); imputability and result of the reaction (recovered, recovered with sequelae, not recovered, unknown, life risk). Ninety-seven patients were registered, of which 26 (27\%) presented adverse events. The most frequently affected organs were the ones in the gastrointestinal system $(14,54 \%)$ and then the ones in the cardiovascular system $(5,19 \%)$. Three of them were severe (1I\%) and required to stop the medication, 7 were moderate $(27 \%)$ and 16 were mild $(62 \%)$. $100 \%$ recovered ad integrum. According to the mechanism of production $22(85 \%)$ were type A and 4 (I5\%) were type B. According to the imputability $18(82 \%)$ were probable and 3 were proved (12\%). The data obtained show a potential for cardiovascular events. This should be taken into account when making decisions related to dental clinical practice in order to avoid unnecessary use.

\section{Key words}

Drug Related Side Effects and Adverse Reactions. Azithromycin. Clarithromycin.

\section{Resumo}

Em Odontologia os antibióticos para o tratamento de processos infeciosos são prescritos empiricamente, sendo os macrólidos uma alternativa terapêutica para casos de hipersensibilidade e resistência bacteriana. No entanto seu uso não está isento de riscos. O objetivo deste estudo foi identificar e caracterizar os eventos adversos associados ao uso de claritromicina e azitromicina. Realizou-se um estudo observacional-descritivotransversal de Farmacovigilância Proativa em pacientes de dois Centros de Saúde da Cidade de Corrientes que foram tratados com antibióticos macrólidos, azitromicina ou claritromicina, por um período de dezoito meses. Variáveis analisadas: idade, sexo, tratamento farmacológico atual de acordo ao diagnóstico, caraterísticas dos eventos adversos identificados segundo: órgãos e tecidos afetados; gravidade, tipo de reação (A ou $B$ de acordo ao mecanismo de produção); imputabilidade e resultado da reação (recuperado, recuperado com sequelas, não recuperado, desconhecido, risco de morte). Registraram-se 97 pacientes, dos quais, 26 (27\%) presentaram eventos adversos. Os órgãos afetados maioritariamente foram os do sistema gastrointestinal $(14,54 \%)$ e cardiovascular, em segundo lugar (5, $19 \%$ ). Três foram graves (1 $1 \%$ ) e requereram a suspensão da medicação, 7 moderados (27\%) e 16 leves (62\%). Recuperaram- se ad integrum $100 \%$. Segundo o mecanismo de produção 22 (85\%) foram do tipo A e 4 (I5\%) do tipo B. Segundo a imputabilidade I 8 (82\%) foram prováveis e 3 provadas ( $12 \%)$. Os dados obtidos mostram um potencial de eventos cardiovasculares, cuja gravidade deveria ser levada em conta no memento de tomar decisões) para tomar decisões relacionadas à prática clínica odontológica a fim de evitar um uso desnecessário. 


\section{Palavras chave}

Efeitos Colaterais e Reações Adversas Relacionadas a Medicamentos. Azitromicina Claritromicina.

\section{Introducción}

En Odontología, la mayoría de las patologías infecciosas son de origen bacteriano y tanto el odontólogo general como las distintas especialidades utilizan antibióticos como complemento de su práctica clínica con la finalidad de erradicar el foco infeccioso y evitar su diseminación a otros tejidos y órganos. Se los usa en forma tópica o sistémica de manera empírica, adaptando la dosis al paciente según la edad, patología y gravedad de la situación clínica; sin considerar parámetros farmacocinéticos (como ser absorción oral, biodisponibilidad, vida media plasmática), resistencia bacteriana, potenciales interacciones y sobre todo aspectos relacionados con la seguridad de estos agentes'.

Los $\beta$ lactámicos, en especial las penicilinas, han sido siempre los antibióticos de primera elección debido a su eficacia y seguridad. Sin embargo, la aparición de cepas multiresistentes y la presencia de reacciones de hipersensibilidad, posicionaron a los antibióticos macrólidos como una alternativa terapéutica en el manejo de las infecciones orales ${ }^{2}$.

Los macrólidos son antibióticos bacteriostáticos que cubren con su espectro de acción a bacterias anaerobias estrictas. El primer antibiótico del grupo o prototipo fue la eritromicina, pero tenía la desventaja de presentar baja biodisponibilidad y toxicidad sobre todo a nivel hepático. Esta situación llevó a que se realicen modificaciones en la estructura química original, sintetizando nuevas moléculas como claritromicina y azitromicina que presentaban mejores propiedades farmacológicas como una rápida absorción, mejor penetración tisular y ampliaban el espectro de acción ${ }^{3}$. Ambos fármacos fueron adquiriendo un lugar en la terapéutica odontológica como alternativa en pacientes alérgicos; en Odontopediatría ${ }^{4}$; para profilaxis de la endocarditis bacteriana 0 profilaxis de infecciones post cirugía de terceros molares impactados ${ }^{5}$ y mostraron un beneficio potencial como adyuvante a la terapia periodontal no quirúrgica en pacientes con periodontitis crónica ${ }^{6,7}$.

La azitromicina posee la ventaja de un régimen de dosificación bastante simple (una dosis diaria) y alcanza concentraciones más altas y sostenidas en el fluido crevicular gingival que en el suero, además de poseer un amplio espectro de acción antimicrobiana hacia las bacterias anaerobias ${ }^{3}$. Este sencillo protocolo de administración tiende a reducir los problemas relacionados con el cumplimiento del paciente.

El uso de estos nuevos macrólidos suponía un menor riesgo de efectos adversos. Sin embargo, la Food and Drug Administration (FDA), emitió un alerta sobre el riesgo de arritmias potencialmente fatales asociado al uso de azitromicina, debido a su capacidad para alterar la actividad eléctrica del corazón ${ }^{8}$. Situación similar se presentó con la claritromicina. En estudios de cohortes prospectivos se observó asociación de claritromicina con eventos cardiovasculares observándose síndrome coronario agudo, insuficiencia cardíaca descompensada, arritmias graves y casos de muerte súbita? ${ }^{9}$.

El conocimiento de estos potenciales efectos secundarios derivados del uso de medicamentos - eventos adversos está íntimamente ligado al ámbito de la Farmacovigilancia.

La Farmacovigilancia es una actividad de Salud Pública destinada a analizar y gestionar los riesgos de los medicamentos una vez comercializados. Se la define como ciencia que se encarga de recopilar, monitorear, investigar, valorar la calidad, y evaluar la información, acerca de los efectos adversos por medicamentos, productos biológicos, así como aquellos empleados en medicina tradicional, buscando identificar información nueva relacionada con las reacciones adversas y prevenir el daño en los pacientes". En Farmacovigilancia se notifican "sospechas" de efectos adversos, es decir eventos adversos por medicamentos (EAM). Pero para poder considerarlas reacciones adversas a medicamentos (RAM), se debe hacer un cuidadoso análisis de causalidad o imputabilidad y establecer si hubo 0 no asociación temporal desde la administración del fármaco hasta la aparición del efecto.

Las RAM son reacciones nocivas, no buscadas y que aparecen con dosis terapéuticas utilizadas en el hombre para la profilaxis, el 
diagnóstico, el tratamiento o la modificación de funciones fisiológicas ${ }^{12}$. Provocan entre $5 \%$ y $10 \%$ de los ingresos hospitalarios y son actualmente entre la cuarta y sexta causa de muerte en países desarrollados ${ }^{13}$.

En Farmacovigilancia existen diversos métodos para identificar EAM, siendo la notificación voluntaria el más utilizado por los países miembros de la Organización Mundial de la Salud (OMS). Es un método simple, económico que consiste básicamente en la comunicación de una "sospecha" de un efecto adverso causado por un determinado medicamento, realizado a través de una ficha amarilla estandarizada y confidencial. Abarca a toda la población y a todos los medicamentos. Se inicia desde el mismo momento de la comercialización. Cualquier persona, no solamente el médico o profesional de salud puede hacer una notificación ${ }^{13}$. Sin embargo, también tiene una gran desventaja: la sub-notificación, que puede deberse a una falla en el reconocimiento de la RAM o simplemente a falta de motivación en completar la ficha.

La Farmacovigilancia puede desarrollarse de manera pasiva (notificaciones internas) o proactiva (seguimiento farmacoterapéutico). La detección, registro, notificación y estudio de los EAM es de fundamental importancia. Implica su incorporación dentro de las posibilidades etiológicas a considerar en el proceso diagnóstico. El objetivo de este estudio fue identificar y caracterizar los eventos adversos asociados al uso de claritromicina y azitromicina.

\section{Materiales y Métodos}

Se realizó un estudio observacional descriptivo, transversal utilizando la metodología de Farmacovigilancia Proactiva, que consiste en obtener información de sospechas de RAM de manera sistemática, realizando un cuidadoso seguimiento de los pacientes que están bajo tratamiento de los fármacos en estudio. Se incluyeron a pacientes de dos Centros de Salud de la Ciudad de Corrientes que recibían alguno de los antibióticos macrólidos: azitromicina o claritromicina, durante un período de dieciocho meses. Se los contactó personalmente o vía telefónica $y$ en caso de sospecha de RAM, se procedió a completar la ficha amarilla de comunicación de
Reacciones Adversas del Centro Regional de Farmacovigilancia de la Universidad Nacional del Nordeste (CRF-UNNE). Las variables analizadas fueron edad, sexo, tratamiento farmacológico actual según diagnóstico, características de los eventos adversos identificados de acuerdo al Diccionario de Reacciones Adversas de la Organización Mundial de la Salud (OMS) ${ }^{14}$ y a la clínica y diagnóstico de Laporte y Capella ${ }^{15}$ según órganos y tejidos afectados; gravedad de la reacción, tipo de reacción ( $A$ ○ $B$ según mecanismo de producción) e información que permitiera establecer imputabilidad o asociación temporal con la toma del fármaco. También se consideró el resultado de la reacción: recuperado, recuperado con secuelas, no recuperado, desconocido, riesgo de vida, malformación, muerte.

Los datos fueron cargados en la base de datos del CRF-UNNE y posteriormente analizados mediante estadística descriptiva, estimando medidas de tendencia central para las variables cuantitativas $y$ tablas de frecuencias para las variables categóricas.

La valoración de la gravedad se realizó, de acuerdo al grado de compromiso que produce la RAM en la salud del paciente, si interfiere con sus actividades habituales, si obliga a efectuar tratamiento farmacológico, hospitalización o prolonga la estadía de la misma, si pone en peligro la vida del paciente, es causa de invalidez, incapacidad, o incluso de muerte ${ }^{15}$.

Para el mecanismo de producción se utilizó la clasificación de Rawlins y Thompson ${ }^{16}$ que clasifica a las RAM en tipo A (Augmented), dependiente de la dosis y del mecanismo de acción del fármaco, predecible; y RAM tipo B (Bizarre), idiosincrásica, no dependiente de la dosis, impredecible.

Para establecer la imputabilidad se utilizó el algoritmo de Karch y Lasagna modificado por Naranjo ${ }^{17}$, que establece en función de cuatro criterios (secuencia temporal, plausibilidad biológica, efecto de retirada, reexposición y causa alternativa) la probabilidad de que un EAM corresponda a una RAM, y la clasifica en dudosa, posible, probable o probada.

Acorde a la Resolución I480/I I del Ministerio de Salud de la Nación "Guía para Investigaciones con Seres Humanos", este trabajo de Investigación constituye un estudio de bajo riesgo, 
por lo que se exceptúa su evaluación por parte de un Comité de Bioética en Investigación.

\section{Resultados}

Se realizó el seguimiento a 97 pacientes que recibieron antibióticos macrólidos, de los cuales 26 (27\%) presentaron EAM. Los diagnósticos que motivaron la prescripción fueron: Neumonía Atípica (8), Enfermedad Pulmonar Obstructiva Crónica (EPOC) reagudizada (6), Neumonía Adquirida de la Comunidad (NAC) (4), Bronquitis (3), Faringitis (2), Sinusitis (I), Infección de Tracto Urinario (ITU) complicada (I). Del total de notificaciones recibidas, 5 (19\%) estuvieron asociadas al uso de Azitromicina y 21 (8I\%) a Claritromicina. En la tabla I se describen las características basales de la población estudiada.

Caracterización de las RAM asociadas a claritromicina y azitromicina

Las RAM afectaron con mayor frecuencia al sistema digestivo $y$ al sistema cardiovascular en segundo término. En la Figura I, se pueden apreciar los órganos y sistemas afectados.

De acuerdo a la gravedad 3 fueron graves (I I\%), 7 moderadas (27\%) y I 6 leves (62\%). Los casos graves eran de pacientes internados y fue necesario suspender el fármaco y prolongar la estadía hospitalaria hasta la remisión de la reacción (58\%). El 100\% de las RAM notificadas se recuperaron ad integrum.

En la tabla II, se describen las RAM graves y moderadas identificadas con los fármacos implicados, resaltándose las que afectaron al sistema cardiovascular.

Según el mecanismo de producción, 22 (85\%) fueron tipo A dependiente de la dosis y 4 (I5\%) fueron por mecanismo de hipersensibilidad (reacciones tipo B). Según la imputabilidad I 8 (82\%) fueron clasificadas como probables y 3 como probadas (I2\%).

\section{Discusión}

En la presente investigación se obtuvo un mayor número de notificaciones de RAM por claritromicina debido en parte a una mayor utilización como fármaco de elección para las neumonías atípicas. Estas reacciones, fueron más frecuentes en las mujeres que en los hombres, lo que sugiere que las mujeres son las que consultan más por problemas de salud coincidente con otras investigaciones ${ }^{18}$.

De 10 RAM moderadas y graves, 5 afectaron al sistema cardiovascular, obligaron a suspender el tratamiento y prolongaron la hospitalización. El Sistema Nacional de Farmacovigilancia (SNFVG) de nuestro país realizó a fines del año 2013 una revisión para conocer el perfil de eventos adversos cardiovasculares asociados al uso de claritromicina y azitromicina, en base a las alertas emitidas internacionalmente. Analizaron un total de 64 notificaciones de eventos adversos asociados al uso de estos macrólidos (20 con azitromicina y 44 con claritromicina) correspondientes al período 2010-2012 y encontraron sólo un reporte de evento adverso cardiovascular (prolongación del QT). La mayoría de las notificaciones recibidas correspondieron a reacciones adversas del tracto gastrointestinal y de la piel similar a lo hallado en esta investigación ${ }^{19}$.

Ponsonnaille, fue el primero en evaluar los efectos electrofisiológicos de un macrólido, eritromicina intravenosa, describió que el efecto sobre el intervalo QT estaba en relación directa con la velocidad de infusión ${ }^{20}$. Varios agentes de la clase de antibióticos macrólidos son conocidos por interferir con la corriente rectificadora tardía de potasio, lo que resulta en acumulación de iones de potasio en los miocitos cardíacos y de ese modo se retrasa la repolarización cardía$\mathrm{ca}$, que se evidencia en el electrocardiograma (ECG) como una prolongación del intervalo QT. La prolongación del intervalo QT y la posibilidad de provocar una arritmia grave subsiguiente, se cree que es un mecanismo de toxicidad cardíaca aguda con macrólidos ${ }^{21}$. En esta investigación se hizo un seguimiento proactivo a los pacientes que recibieron azitromicina y claritromicina y se halló cuatro eventos cardiovasculares asociados a claritromicina y uno a azitromicina. Estos datos no permiten afirmar que la azitromicina tenga menor riesgo tal como sugieren algunas investigaciones $^{22}$. Es necesario continuar con la vigilancia epidemiológica a largo plazo para tener datos más precisos. 
Tabla I. Características basales de la Población en estudio.

Características de la población

$\mathrm{n}=97$

\begin{tabular}{lccc} 
& Media & DS & Rango \\
\hline Edad (años) & 48 & 19 & $7-79$ \\
\hline Total de EAM notificados & n & $\%$ & 27 \\
\hline Total EAM por Sexo: femenino & 26 & 58 \\
\hline EAM notificadas por Claritromicina & 15 & 81 \\
\hline EAM notificadas por Azitromicina & 21 & 19 \\
\hline RAM más frecuentes: Gastrointestinales & 5 & 54
\end{tabular}

Tabla II. Descripción de las RAM graves y moderadas notificadas en relación a los medicamentos sospechados

\begin{tabular}{|c|c|c|c|c|c|c|c|}
\hline 导 & 齐 & 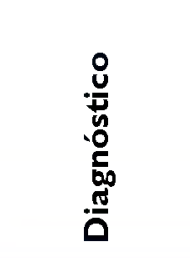 & 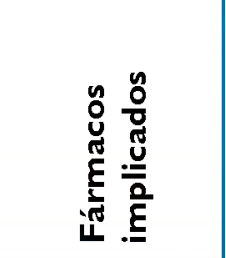 & 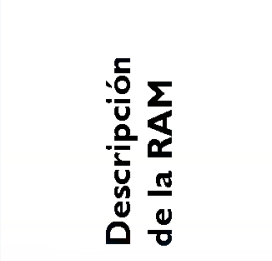 & 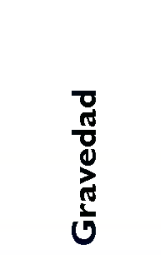 & 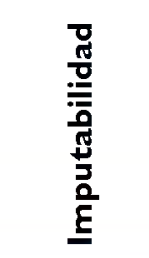 & 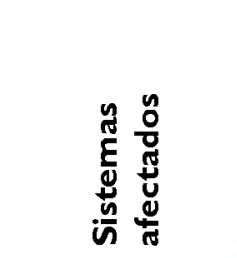 \\
\hline 65 & $M$ & $\begin{array}{l}\text { EPOC } \\
\text { reagudizado }\end{array}$ & Claritromicina & $\begin{array}{l}\text { Taquicardia } \\
\text { Ventricular }\end{array}$ & Grave & Probable & $\begin{array}{c}\text { Sistema } \\
\text { cardiovascular }\end{array}$ \\
\hline 62 & $M$ & $\begin{array}{l}\text { EPOC } \\
\text { reagudizado }\end{array}$ & Claritromicina & $\begin{array}{l}\text { Taquicardia } \\
\text { Ventricular }\end{array}$ & Grave & Probable & $\begin{array}{c}\text { Sistema } \\
\text { cardiovascular }\end{array}$ \\
\hline 35 & $\mathrm{~F}$ & NAC grave & Claritromicina & $\begin{array}{l}\text { Taquicardia } \\
\text { Ventricular }\end{array}$ & Grave & Probable & $\begin{array}{c}\text { Sistema } \\
\text { cardiovascular }\end{array}$ \\
\hline 48 & $\mathrm{~F}$ & Bronquitis & Azitromicina & Taquicardia & Moderada & Probable & $\begin{array}{c}\text { Sistema } \\
\text { cardiovascular }\end{array}$ \\
\hline 78 & $F$ & $\begin{array}{l}\text { EPOC } \\
\text { reagudizado }\end{array}$ & Claritromicina & $\begin{array}{l}\text { Extrasístole } \\
\text { Ventricular }\end{array}$ & Moderada & Posible & $\begin{array}{c}\text { Sistema } \\
\text { cardiovascular }\end{array}$ \\
\hline II & $M$ & $\begin{array}{l}\text { Neumonía } \\
\text { atípica }\end{array}$ & Claritromicina & $\begin{array}{c}\text { Diarrea. } \\
\text { Dispepsia.Cefalea }\end{array}$ & Moderada & Probable & $\begin{array}{c}\text { Sistema } \\
\text { Gastrointestinal } \\
\text { y nervioso }\end{array}$ \\
\hline 79 & $\mathrm{~F}$ & Faringitis & Azitromicina & Diarrea & Moderada & Probada & $\begin{array}{c}\text { Sistema } \\
\text { Gastrointestinal }\end{array}$ \\
\hline 46 & $\mathrm{~F}$ & NAC & Claritromicina & $\begin{array}{l}\text { Elevación de } \\
\text { Transaminasas }\end{array}$ & Moderada & Probable & $\begin{array}{l}\text { Trastornos } \\
\text { del hígado }\end{array}$ \\
\hline 7 & $F$ & $\begin{array}{c}\text { Neumonía } \\
\text { atípica }\end{array}$ & Claritromicina & $\begin{array}{l}\text { Rash generalizado } \\
\text { al } 5^{\circ} \text { día de } \\
\text { administración }\end{array}$ & Moderada & Probable & Piel y anexos \\
\hline 45 & $F$ & $\begin{array}{c}\text { Neumonía } \\
\text { Atípica }\end{array}$ & Claritromicina & $\begin{array}{l}\text { Elevación de } \\
\text { Transaminasas }\end{array}$ & Moderada & Probable & $\begin{array}{l}\text { Trastornos } \\
\text { del hígado }\end{array}$ \\
\hline
\end{tabular}


Figura I. Órganos y sistemas afectados por EAM.

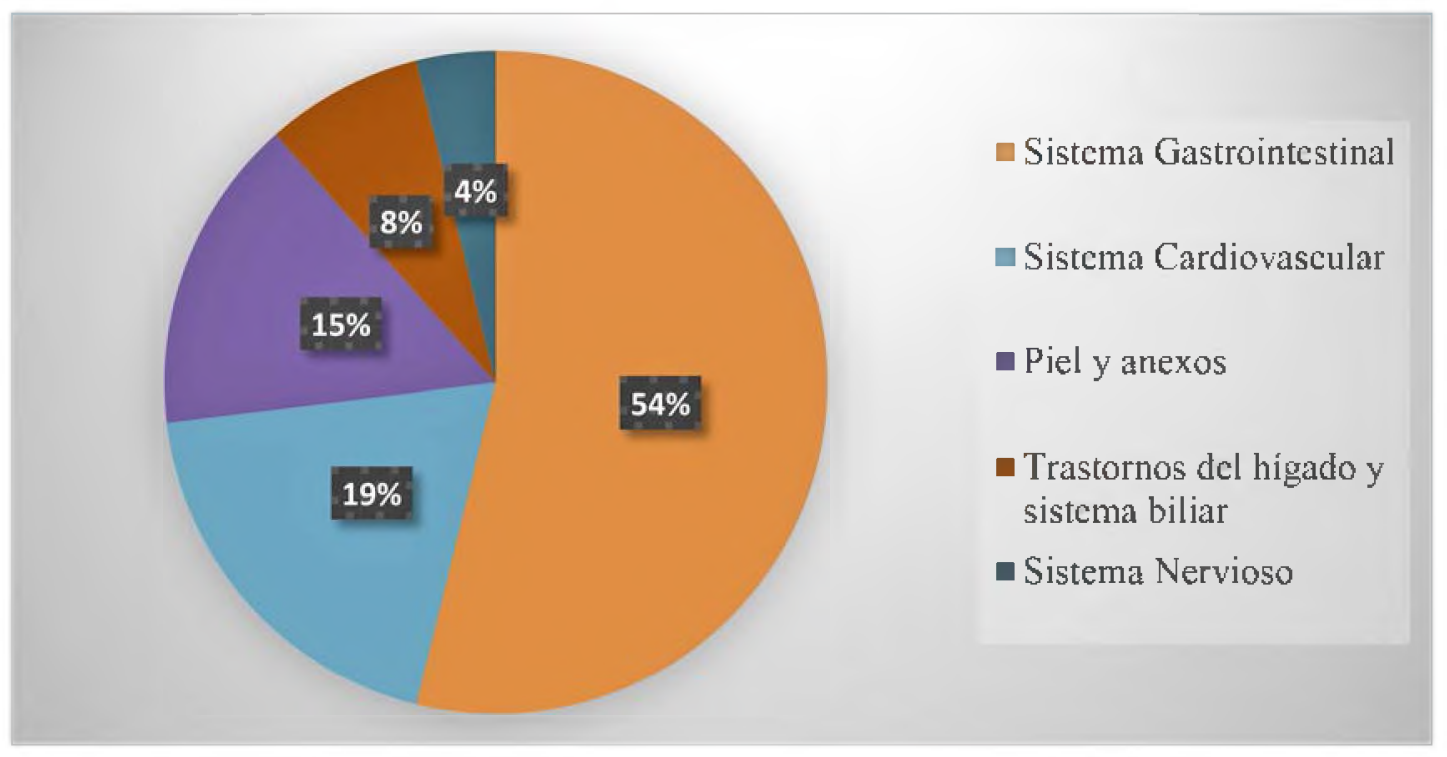

La mayoría de las RAM fueron catalogadas como probables, coincidente con el informe emitido por el SNFVG, es decir, que dichos eventos no podrían ser explicados por una causa alternativa como ser la patología de base del paciente, alguna interacción farmacológica $\circ$ algún otro factor ${ }^{19}$.

Es importante destacar que existen controversias en relación a si estos fármacos pueden producir per se un aumento de riesgo de eventos cardiovasculares o si el mismo puede explicarse por la presencia de factores de riesgos previos en los pacientes o por interacción con otros fármacos que también producirían un incremento en el intervalos QT. Ambos fármacos son considerados como categoría de riesgo I dentro de un listado, pertenecientes a sustancial evidencia de que estos fármacos pueden prolongar el intervalo QT y tienen un riesgo de producir torsada de punta ${ }^{23}$.

En un estudio de eventos cardiovasculares, registrados en Buenos Aires-Argentina se encontró que sobre un total de 2516 notificaciones, $|5|$ reacciones adversas afectaban el aparato cardiovascular, de las cuales 22 (14,6\%) fueron graves, incluyendo 6 afecciones potencialmente mortales. Hubo 3 casos de paro cardíaco (que se produjo durante procedimientos anestésicos), sin consecuencias fatales. Los fármacos implicados fueron antimicrobianos del grupo de los macrólidos $(27,2 \%)$ y fármacos neurológicos $(21,2$ $\%)$. Las RAM más frecuentes informados fueron síndrome de QT largo 58 (38,4\%), edema periférico I5 (9,9\%) e hipotensión I5 (9,9\%) ${ }^{24}$.

También se identificaron reacciones moderadas, a nivel de otros sistemas como la piel (reacciones de hipersensibilidad), a nivel del sistema hepático y nervioso que llaman la atención porque estos nuevos macrólidos tienen un mejor perfil de seguridad en relación a su prototipo, eritromicina, sin embargo, esos efectos descriptos, son un efecto de clase o grupo, por lo tanto no están exentos de producirlos ${ }^{25}$.

La mayoría de las RAM fueron de tipo $A$, es decir debidas a un efecto aumentado en relación al mecanismo de acción. Por ejemplo, los efectos secundarios sobre el aparato digestivo que fueron los más referidos, se deben a que los macrólidos actúan como antagonistas sobre los emisores de la motilina $y$, en consecuencia, 
REVISTA FACULTAD DE ODONTOLOGIAA

ISSN N ${ }^{\circ} 1668-7280$ - Vol. XI N ${ }^{\circ} 1-2018$

14

INVESTIGACION

Rocha - Morales - Harman - Schmidt - Servin - Dos Santos estimulan la motilidad gastrointestinal. Este tipo de reacciones pueden ser evitadas, ajustando la dosis del fármaco.

\section{Conclusiones}

Los datos de este estudio, permiten brindar información más actualizada acerca de los riesgos asociados al uso de azitromicina y claritromicina, dos fármacos que están ganando lugar en la terapéutica odontológica. Su perfil muestra un potencial de eventos cardiovasculares que, de acuerdo a su gravedad $y$ a los informes emitidos por las agencias reguladoras, habría que tenerlos en cuenta en la toma de decisiones relacionadas con la práctica clínica odontológica a fin de evitar un uso innecesario. Demuestra que las RAM afectan la calidad de vida de los pacientes, generan pérdida de confianza en el prescriptor, aumentan los costos sanitarios, implican un cambio en el tratamiento y además pueden imitar enfermedades, dificultando su detección. Todos estos factores debieran tener en cuenta los odontólogos a la hora de prescribir un fármaco para evitar exponer al paciente a riesgos potenciales que afecten a su salud.

\section{Referencias Bibliográficas}

I. Herrera Ustariz, Iván. Azitromicina: un macrólido diferente: Perfil terapéutico en infecciones. Rev Fed Odontol Colomb. 2005; (23): I-8.

2. Bascones Martínez A, Aguirre Urízar JM, Bermejo Fenoll A, Blanco Carrión A, GayEscoda C, González Moles MA et al. Documento de consenso sobre el tratamiento antimicrobiano de las infecciones bacterianas odontogénicas. Av Odontoestomatol [Internet]. 2005 Dic [citado 2018 Nov 06]; 2I(6):3| |-3|9. Disponible en: http://scielo. isciii.es/pdf/odonto/v2 I n6/original3.pdf

3. Millones-Gómez P, Aguilar A. Eficacia de la Azitromicina asociada al raspado y alisado radicular en periodontitis crónica. Rev. Clin. Periodoncia Implantol. Rehabil. Oral [Internet]. $2017 \mathrm{Abr}$ [citado 2018 Nov 06]; I0(I):3337. Disponible en: https://scielo.conicyt.cl/ scielo.php?script=sci_arttext\&pid=s0719. $01072017000100033 \&$ Ing=es. http://dx.doi. org/10.4067/S07/9-01072017000100033

4. Pimentel Herrezuelo E, Salazar de Plaza E. Uso de azitromicina en odontopediatría. Acta Odontol Venez. 200 I; 39 (2): 64-69.

5. Salmerón Escobar Jl, Amo Fernández de Velasco A del. Profilaxis antibiótica en $\mathrm{Ci}$ rugía Oral y Maxilofacial. Med. oral patol. oral cir.bucal (Internet) [Internet]. 2006 Jun [citado 2018 Nov 07]; II(3): 292296. Disponible en: http://scielo.isciii.es/ scielo.php?script=sci_arttext\&pid=S1698$69462006000300016 \&$ Ing=es.

6. Buset SL, Zitzmann NU, Weiger R, Walter C. Non-surgical periodontal therapy supplemented with systemically administered azithromycin: a systematic review of RCTs. Clin Oral Investig. 20I5; 19 (8): I763-75.

7. Zhang Z, Zheng $Y$, Bian X. Clinical effect of azithromycin as an adjunct to non-surgical treatment of chronic periodontitis: a metaanalysis of randomized controlled clinical trials. J Periodontal Res. 2016; 5 I (3): 275-83. 
8. FDA Drug Safety Communication: Azithromycin (Zithromax or Zmax) and the risk of potentially fatal heart rhythms, (20I3).

9. Schembri S, Williamson PA, Short PM, Singanayagam A, Akram A, Taylor J, et al. Cardiovascular events after clarithromycin use in lower respiratory tract infections: analysis of two prospective cohort studies. BMJ. Mar 20;346:f| 235. doi: |0.। | 36/bmj.f|235.

10. Ray WA, Murray KT, Hall K, Arbogast PG, Stein CM. Azithromycin and the risk of cardiovascular death. NEJM. 20I2; 366 (20): | 88|-90.

II. Organization WH. World alliance for patient safety : WHO draft guidelines for adverse event reporting and learning systems: from information to action. 2005.

12. Organization WH. Marco Conceptual de la Clasificación Internacional para la Seguridad del Paciente Versión I 2009. p. 160.

13. Valsecia M. Farmacovigilancia y mecanismos de reacciones adversas a medicamentos. Farmacología Médica. 2000; 5: I 35-48.

14. International monitoring of adverse drug reactions. WHO Collaborating Centre for International Drug Monitoring. Ginebra: OMS, 1988.

15. Laporte J, Capellà D, Laporte J, Tognoni G. Mecanismos de producción y diagnóstico clínico de los efectos indeseables producidos por medicamentos. Principios de epidemiología del medicamento. 1993; 2.

16. Rawlins MD T]. Mechanisms of adverse drug reactions. In: DM. D, editor. Textbook of Adverse Drug Reaction. 4 ed. Oxford 1991. p. 18-45.

17. Naranjo CA BU, Sellers EM, Sandor P, Ruiz I, Roberts EA, Janecek E. A method for estimating the probability of adverse drug reactions. Clin Pharmacol Ther. 1981; 30: 239-45.
18. Svanström H, Pasternak B, Hviid A. Use of clarithromycin and roxithromycin and risk of cardiac death: cohort study. BMJ. 2014 2014-08-19 22:30: 59; 349.

19. Administración Nacional de Medicamentos, Alimentos y Tecnología Médica (ANMAT). Novedades Internacionales y $\mathrm{Na}$ cionales en Seguridad de Medicamentos, Septiembre 2013. Disponible en: http:// www.anmat.gov.ar/farmacovigilancia/Novedades_FVG_septiembre_20।3.pdf

20. Ponsonnaille J, Citron B, Richard A, Trolese JF, Chaperon A, Barret B, et al. Electrophysiological study of pro-arrhythmogenic effects of erythromycin Arch Mal Coeur Vaiss. 1988; 8I: 1001-8.

2 I. Albert RK, Schuller JL. Macrolide antibiotics and the risk of cardiac arrhythmias. AJRCCM. 2014; 189 (10): II73-80.

22. Milberg P, Eckardt L, Bruns H-J, Biertz J, Ramtin S, Reinsch N, et al. Divergent proarrhythmic potential of macrolide antibiotics despite similar QT prolongation: fast phase 3 repolarization prevents early afterdepolarizations and torsade de pointes. JPET. 2002; 303 (I): 2 I8-25.

23. Hayashi $Y$, Ikeda $U$, Hashimoto $T$, Watanabe T, Mitsuhashi T, Shimada K. Torsades de pointes ventricular tachycardia induced by clarithromycin and disopyramide in the presence of hypokalemia. PACE. 1999; 22 (4): 672-4.

24. Keller GA DGG, Alvarez PA. Pharmacovigilance and the cardiovascular system: two sides to every story. Curr Drug Saf. 201 I; 6 (4): 224-9.

25. Robles M, Andrade RJ. Hepatotoxicidad por antibióticos: actualización en 2008. Rev Esp Quimioter 2008; 2 I (4): 224-233.

Los autores declaran no poseer conflicto de intereses.

Trabajo financiado por la Secretaría General de Ciencia y Técnica de la Universidad Nacional del Nordeste. 